\title{
Índice de ahorro de tiempo medio de viaje cómo variable complementaria en la metodología Contribución por Valorización para la financiación de infraestructura vial
}

\author{
Santiago Cardona1, Diego A. Escobar ${ }^{1 *}$ y Carlos A. Moncada ${ }^{2}$ \\ (1) Universidad Nacional de Colombia, Sede Manizales. Facultad de Ingeniería y Arquitectura, Departamento de \\ Ingeniería Civil, Grupo de Investigación en Movilidad Sostenible. Carrera 37 con Calle 94, Campus La Nubia, Manizales, \\ 170003, Colombia. (correo-e: scardonau@unal.edu.co; daescobarga@unal.edu.co) \\ (2) Universidad Nacional de Colombia, Sede Bogotá. Facultad de Ingeniería, Departamento de Ingeniería Civil y \\ Agrícola, Programa de Investigación en Tránsito y Transporte - PIT. Ciudad Universitaria edificio 214 oficina 417 , \\ Bogotá, 111321, Colombia. (correo-e: camoncadaa@unal.edu.co)
}

* Autor a quien debe ser dirigida la correspondencia.

Recibido Sep. 23, 2019; Aceptado Nov. 18, 2019; Versión final Ene. 3, 2020, Publicado Ago. 2020

\section{Resumen}

En este artículo de investigación se propone el índice de ahorro cómo variable complementaria en el cálculo de la asignación del cobro por Contribución por Valorización usado para financiar proyectos de infraestructura vial a través del modelo Manizales. Se propone utilizar la accesibilidad media global y accesibilidad media integral para medir los tiempos de viaje en distintos escenarios, cuantificar el ahorro generado por el proyecto propuesto y complementar dos variables de movilidad expuestas en los modelos de asignación de recaudo. La accesibilidad media global calcula el ahorro dado por el proyecto mientras que la accesibilidad media integral mide la mejora en tiempo de viaje generada por el proyecto. Se encontró que el modelo Manizales aplicado para financiar obras mediante el cobro por Contribución por Valorización posee una mayor aceptación entre los habitantes donde se aplicó el cobro y se concluye que puede ser complementado adecuadamente al involucrar las variables propuestas.

Palabras clave: accesibilidad; contribución por valorización; modelos de transporte; índice de ahorro

\section{Average travel time saving index as a complementary variable in the methodology Betterment Levy for financing road infrastructure}

\begin{abstract}
This research article proposes the saving index as a complementary variable for the calculation of the allocate benefit in the financing of road infrastructure projects by betterment levy in the Manizales model. For this purpose, it is proposed to use the global accessibility average and the integral accessibility average to calculate travel times on different scenarios, to quantify the savings generated by the proposed project, and to complement two mobility variables presented in the betterment levy models for benefit allocation. The global accessibility average calculates the savings generated by the project. The integral accessibility average measures the improvements on travel times generated by the project. The results show that the Manizales model that was applied to financing projects through betterment levy is best accepted by inhabitants where taxes were raised. In conclusion, the proposed variables can be applied to improve and complement the Manizales model.
\end{abstract}




\section{INTRODUCCIÓN}

Con el objetivo de mejorar las condiciones de movilidad en el área urbana y rural de las ciudades, a través de los años se han impulsado grandes obras de infraestructura vial tal cómo puentes, intercambiadores viales, túneles, entre otras, que generan un gasto importante en el presupuesto de las regiones por lo que evaluar el orden de prioridad del gasto y la forma cómo se financian dichas obras ha obtenido gran relevancia en las últimas décadas (Escobar y García, 2012; Miramontes et al., 2015). En este sentido, los modelos de transportes se han convertido en un arma fundamental para evaluar el impacto generado por las obras de infraestructura vial mediante los modelos de oferta y demanda del transporte (Ortúzar y Willumsen, 2011; Owens, 1995), mientras que la Contribución por Valorización (CV) ha sido un instrumento para financiar dichas obras en las ciudades alrededor del mundo, generando un cobro por el mejoramiento a los inmuebles privados que genera la construcción de un bien público (Fernández, 1981; Navarro, 2016). En América Latina y el Caribe, la recuperación de plusvalías ha sido un tema con el cual los entes gubernamentales han estado familiarizados, sin embargo, solo algunos países poseen legislación nacional que les permite aplicarla, siendo Colombia y Brasil los países de la región donde más frecuentemente se utilizan (Smolka, 2012). Por otra parte, al momento de establecer el cobro por CV los países se encuentran con obstáculos de tipo legislativo, la necesidad de establecer un fundamento al cobro, las dificultades técnicas de su implementación, el riesgo político y la falta de información (Smolka, 2012). En Colombia, la CV ha sido frecuentemente utilizado desde el siglo pasado con el fin de financiar la construcción de obras de infraestructura urbanas y rurales las cuales deben tener la característica de generar beneficios específicos e incremento en el valor de la propiedad de su área de influencia (Borrero, 2011; Navarro, 2016). Dado el éxito que esta CV ha tenido en Colombia, se escogieron sus metodologías para continuar con el debate alrededor de este impuesto.

Para financiar obras a través del cobro por CV, Colombia ha implementado dos metodologías según las ciudades donde se ha desarrollado e implementado: el modelo Bogotá y el modelo Manizales (también conocido cómo modelo Medellín). Ambos deben cumplir con la ley nacional que estipula el cálculo de la CV según el menor entre tres parámetros: el costo de la construcción del proyecto, el valor añadido que genera a los propietarios la construcción del proyecto y la capacidad de pago de los propietarios (Borrero, 2011; Borrero, 2013). El modelo Bogotá es administrado por el Instituto de Desarrollo Urbano (IDU), el cual define un área de influencia según los beneficios generados por el proyecto a financiar. Los beneficios resultantes del proyecto se calculan por zonas, teniendo en cuenta factores cómo: mayor movilidad (aumento de velocidades de operación, menor tiempo de viaje, costos de operación menores, mejor calidad de vida), mejora en la planificación urbana de la red vial y el espacio público, cambios generados en el uso del suelo, mayor valor mercantil de los inmuebles, integración del proyecto en la estructura de la ciudad, recuperación de áreas deterioradas, entre otras (Borrero, 2011). Por otra parte, el modelo Manizales, administrado por el Instituto de Valorización de Manizales (INVAMA) utiliza el modelo del doble avalúo, el cual tiene en cuenta factores socioeconómicos para determinar el área de influencia del proyecto a través del cual se calculan los parámetros para el cobro de la CV entre el efecto de valorización generado por el proyecto y la capacidad de pago de los contribuyentes (personas a las cuales se les cobraría el gravamen siendo quienes se encuentran en la lista de la administración municipal como dueños de predios que se localizan dentro del área de influencia directa o indirecta del proyecto). Para determinar el beneficio generado por el proyecto se lleva a cabo el método del doble avalúo, generándose el mapa de isoprecios sobre el área de influencia. Este método tiene en cuenta puntos sobre el área de influencia (la cual es definida según el impacto dado por el proyecto a la movilidad) donde se realiza una valoración inicial sobre el uso del suelo y se compara con su valor teniendo en cuenta la construcción y el funcionamiento total del proyecto, para lo cual se realizan análisis expost donde se tienen en cuenta proyectos ya realizados donde se realizaron análisis de impacto del uso del suelo (Borrero, 2011; Borrero, 2013). Para estimar el valor añadido que el proyecto genera se llevan a cabo tres principales estudios: i) Económico, el cual define los parámetros que serán calificados en las fórmulas matemáticas de valor añadido; ii) Urbanístico, el cual mide el potencial de cambio de usos del suelo que el proyecto puede llegar generar; y iii) Movilidad, en el cual se estudia la red vial para cuantificar y cualificar el beneficio, midiendo la reducción en distancia de viaje para la población sobre el área de influencia (Borrero, 2011). Luego se distribuye el beneficio calculado teniendo en cuenta unos pesos dados: cambio en el uso del suelo (40\%), mayor acceso a áreas comerciales (20\%), ahorro en el tiempo de viaje, claramente determinado en tiempo y distancia (20\%) y reducción en la congestión y/o contaminación sobre el área de influencia (20\%); posteriormente se establece el nivel de beneficio, teniendo en cuenta el punto focal cómo el área de mayor mejoramiento sobre el área de influencia según todos los factores antes mencionados. Desde este punto se realiza una distribución sobre el mapa de isoprecios antes mencionado. Por último, el INVAMA distribuye el CV teniendo en cuenta la capacidad de pago y se determina un periodo para la recolección del recaudo. La gran diferencia entre estos métodos, además de la metodología aplicada, son los altos niveles de satisfacción percibida entre los usuarios del modelo Manizales, dada la finalización de los proyectos en su plazo establecido o con poco tiempo de retraso, caso contrario al dado en el modelo Bogotá en los últimos años (Borrero, 2011). 
Teniendo en cuenta el nivel de satisfacción y la metodología antes expuesta, se optó en esta investigación, el estudiar el modelo Manizales. El objetivo de esta investigación es el proponer una metodología para calcular las variables que impactan la movilidad: mayor acceso a áreas comerciales y ahorro en el tiempo medio de viaje, las cuales alcanzan un peso del $20 \%$ cada una entre los parámetros del doble avalúo. Para la primera variable se propone utilizar la accesibilidad media integral, medida entre el área de influencia del proyecto y la zona de la ciudad hacia donde más viajes se generan, zona obtenida a través de la matriz origen destino (vigencia menor a 5 años) o mediante la aplicación de encuestas de preferencias declaradas sobre la zona de influencia, mientras que para la segunda variable se propone la utilización de la accesibilidad media global. En ambos casos se propone realizar una comparación entre los escenarios de movilidad actual y futuro, midiendo el impacto a través del gradiente de ahorro y obteniendo el índice de ahorro (IA) por zona, lo que permitirá brindar una mayor precisión al análisis.

La accesibilidad es un modelo de oferta del transporte que mide el potencial de oportunidades de interacción que poseen los individuos en una región (Hansen, 1959). A través de los años, los investigadores y planificadores de ciudad han desarrollado diferentes enfoques de la medida generando distintos tipos cómo la accesibilidad basada en gravedad, en oportunidades, en infraestructura, entre otras (Geurs y Van Wee, 2004). En este caso se utilizará la accesibilidad basada en infraestructura debido a que usa la red de infraestructura vial y sus características operativas representadas a través de la velocidad de operación para determinar su calidad cómo representación pura de la oferta del transporte (Añez et al., 1996). Asimismo, se usan dos tipos de medida: la accesibilidad media global y la accesibilidad media integral. La accesibilidad media global es un promedio de los tiempos de viaje entre todos los nodos de la red vial (Moncada et al., 2018; Talen y Anselin, 1998), mientras que la accesibilidad media integral es una medida desde nodos específicos de la ciudad hacia destinos particulares de la misma o viceversa (Ingram, 1971). En ambos casos se pueden tomar dos enfoques diferentes, presunción de origen o destino y se determinan según el análisis que se desea realizar (Cardona, 2018; Pirie, 1979).

El gradiente ahorro es una metodología que ha sido utilizada en investigaciones previas para medir el impacto generado por distintas obras de infraestructura vial tales cómo el complemento del anillo del sistema vial urbano de Manizales (Moncada et al., 2018), el impacto a la accesibilidad de los habitantes de Bogotá generado por el sistema integrado de transporte público (Guzmán et al., 2018) y por el sistema de buses de tránsito rápido (BRT) en Lima (Oviedo et al., 2019). También, se ha usado para calcular la mejora en la accesibilidad geográfica de la educación superior en Irlanda generada por una serie de reformas (Walsh et al., 2017). Está metodología ha evaluado el impacto a nivel urbano de distintos proyectos y reformas, sin embargo, no se tiene referencia de su uso como complemento de las metodologías para el cobro de CV con fines de financiamiento de proyectos de infraestructura vial.

Con el objetivo de presentar la metodología de cálculo de las variables antes mencionadas, en esta investigación se aborda el proyecto de infraestructura vial "Intercambiador La Carola" en la ciudad de Manizales, financiado en un $75 \%$ a través del cobro de CV a cerca de 15.311 contribuyentes distribuidos en 15 barrios, siendo US $\$ 9$ millones el costo total estimado por la entidad recaudadora (INVAMA, 2017). En la figura 1, se observa la localización geográfica de la ciudad de Manizales la cual posee cerca de 400 mil habitantes y está ubicada sobre una topografía abrupta $(2153 \mathrm{msnm})$ haciendo que sus procesos de construcción y consolidación sean difíciles y costos. Asimismo, se observa la ubicación del proyecto objeto de estudio y el área de influencia previamente definida por el INVAMA para el cobro de la CV. Ahora bien, teniendo en cuenta que la estratificación social en Manizales va numerada del 1 al 6 según la capacidad económica del barrio o sector, siendo el estrato 1 zonas de baja capacidad económica y el estrato 6 zonas de alta capacidad económica, información que es recolectada y actualizada periódicamente por la propia administración municipal. Entre los contribuyentes, el $58 \%$ pertenecen al estrato 3 y sumado con el porcentaje del estrato 4 alcanza el $64 \%$ para el estrato medio, siendo el que mayor influencia tiene. Luego siguen los estratos bajos, con un $25 \%$ para el estrato 2 y una participación menor al $1 \%$ para el estrato 1 . Por último, los estratos altos tienen un porcentaje del $11 \%$, con el estrato 5 en $9.5 \%$.

Luego de la Introducción, se presenta la metodología llevada a cabo en la investigación, posteriormente se presentan los Resultados y Discusión de los mismos, para luego pasar a una Discusión Final y las principales Conclusiones obtenidas.

\section{METODOLOGÍA}

En la figura 2 se observa el diagrama de flujo de la metodología empleada el cual posee seis etapas consecutivas.

Etapa 1. Definición del área de estudio. Cómo área de estudio se define la dispuesta por la entidad encargada de generar el cobro de la CV en cada ciudad según el proyecto a evaluar. En este caso, el INVAMA para el 
cobro de la CV para la construcción de la "Intersección La Carola". En está, incluyeron 15 barrios, que alcanzan un área de 233 hectáreas y aproximadamente 47000 habitantes. Asimismo, indican que el cobro se efectuará a 15.311 contribuyentes. Por otra parte, se extrae del polígono de barrios de la ciudad los barrios del área de influencia con el fin de realizar análisis específicos sobre ellos.

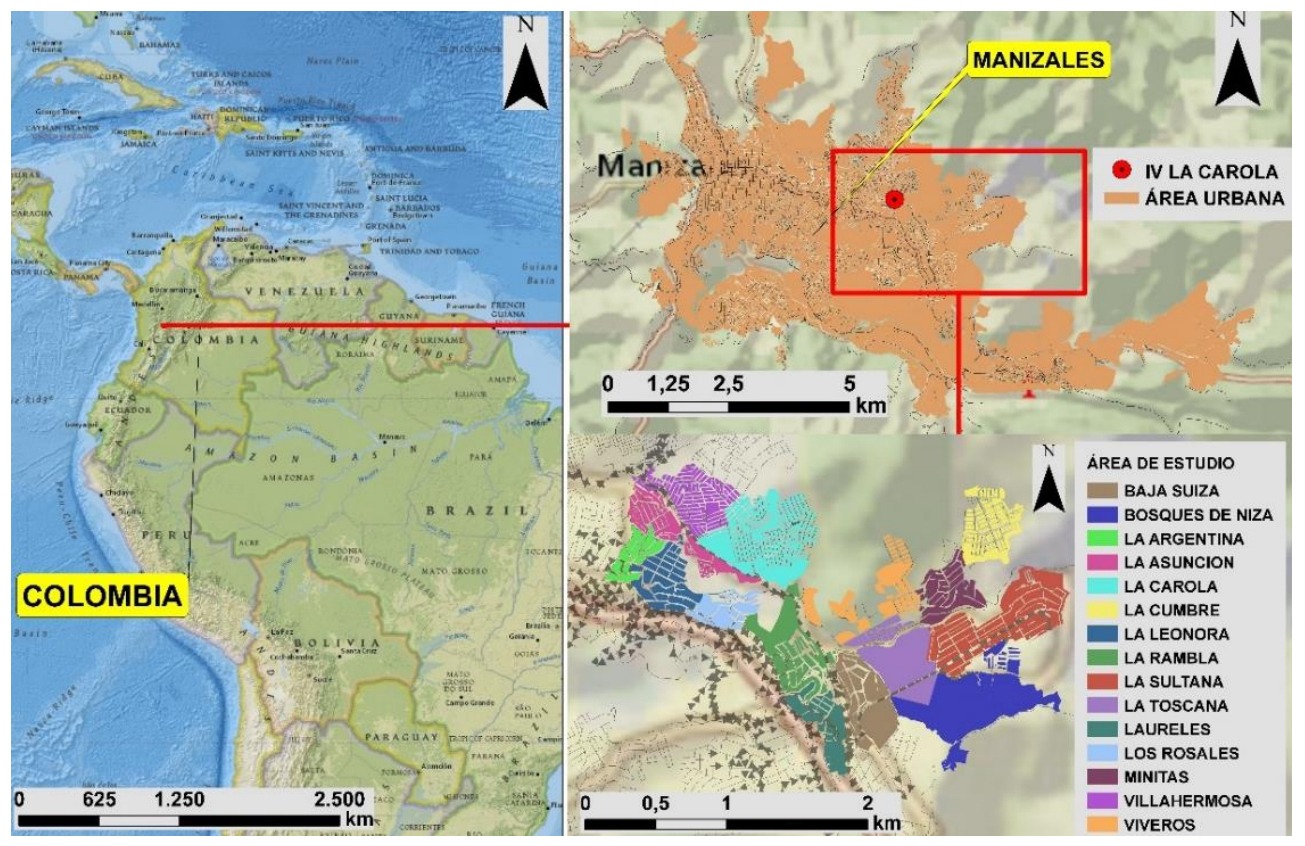

Fig. 1: Localización geográfica del área de estudio en Manizales.

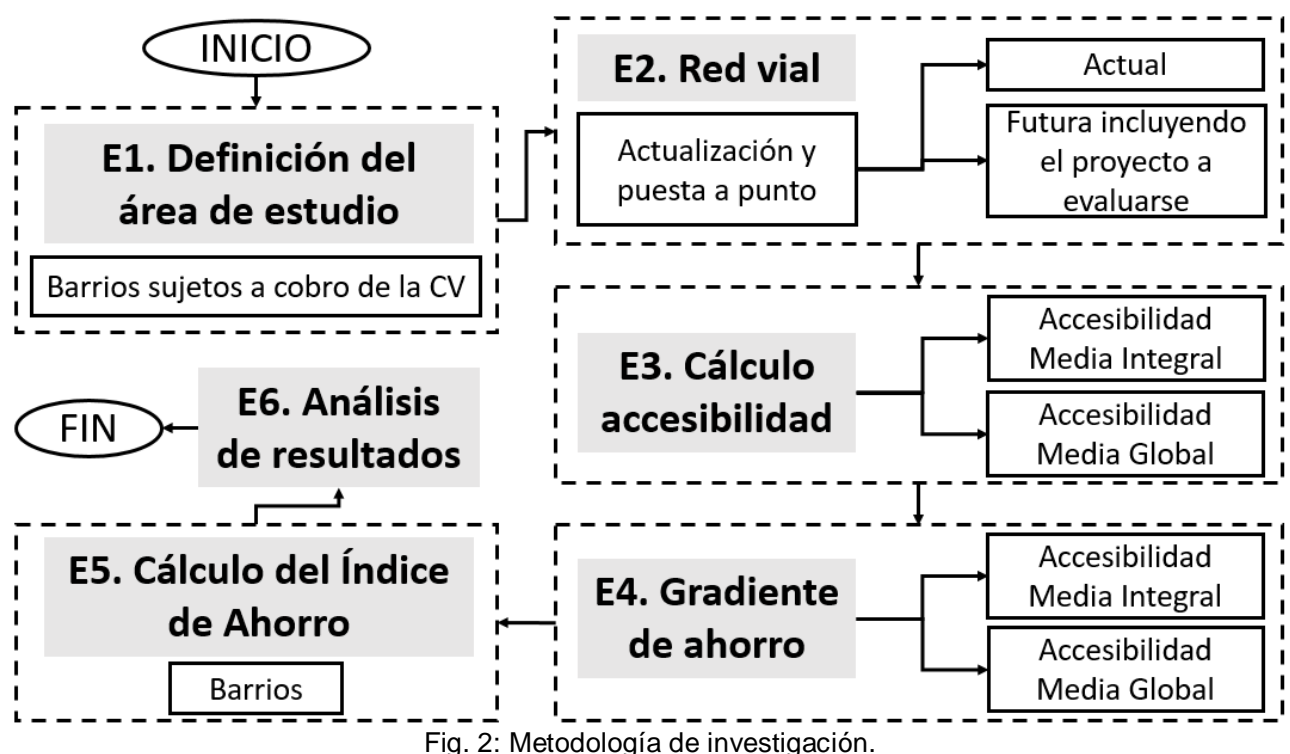

Etapa 2. Red vial. Está etapa de la investigación permite actualizar y poner a punto la red vial de la ciudad, la cual será utilizada para calcular la accesibilidad media global e integral para los escenarios actual y futuro. La red vial actual puede ser obtenida a través de entidades oficiales, construida mediante software SIG o descargada a través de openstreemap. La red debe cumplir con la teoría de grafos, la cual evalúa su conectividad a través de la evaluación de nodos (intersecciones viales) y arcos (vías) (Weber, 2016). También, la red vial debe contar con atributos mínimos de longitud y velocidad. La longitud de los arcos puede ser medida en campo o mediante herramientas incluidas en paquetes de software SIG. Por su parte, la velocidad puede ser definida mediante la utilización de GPS, trabajo de campo de análisis de velocidades punto o la extracción a través de APIS de aplicaciones como Google, Uber, entre otras (Cardona, 2018; Lahoorpoor y Levinson, 2020); todo lo anterior permitirá que la investigación pueda ser replicada en otras regiones 0 ciudades. Por otra parte, para la red vial futura, se incluye el proyecto de infraestructura a evaluarse. Para el caso de estudio, se obtiene la red vial para la situación actual (Moncada et al., 2018), actualizándose con las modificaciones realizadas previa inauguración de la "Intersección La Carola", la cual se realizó en febrero de 2019. En segundo lugar, se ajustó la red de cálculo incluyendo el proyecto de infraestructura vial. La red vial utilizada para la situación actual fue obtenida gracias a investigaciones previas, para la cual se calcularon 
velocidades mediante GPS y las penalidades por giro mediante trabajo de campo (Cardona, 2018). Por otra parte, las velocidades del intercambiador vial se determinaron en $60 \mathrm{~km} / \mathrm{h}$, límite de velocidad para zonas urbanas en Colombia y método utilizado para modelos similares en Alberta, Canadá (Yiannakoulias et al., 2013). Para el cálculo de la accesibilidad se debe tener en cuenta el tiempo de viaje por arco, para lo cual se utiliza el valor de velocidad y longitud de cada uno de ellos (Liu y Zhu, 2004).

Etapa 3. Cálculo accesibilidad. Para la accesibilidad media global se tiene en cuenta la red vial en ambos escenarios, calculando el tiempo de viaje mínimo (tvi) entre todos los nodos de la red (n) cómo origen de los mismos (Pirie, 1979) y obteniendo la matriz de tiempos de viaje mínimo de la red. Para ello se usa el algoritmo Dijkstra (1959), el cual minimiza el tiempo de viaje entre nodos, teniendo en cuenta las restricciones y penalidades por giro de la red (Caldwell, 1961; Cardona, 2018; Delling et al., 2009). Luego, de la matriz de tiempos de viajes mínimos se extrae el vector de tiempo promedio de viaje (TVI), a través de la ecuación 1. Donde el numerador representa la sumatoria del tiempo mínimo de cada nodo de la red vial hacia los demás, mientras el denominador representa el total de nodos a los cuales viaja cada nodo, siendo en este caso el total de nodos de la red vial excepto a él mismo.

$$
\overline{T_{v l j}}=\frac{\sum_{i=1}^{n} t_{v i j}}{(n-1)} \quad i=1,2,3, \ldots, n ; j=1,2,3, \ldots, \mathrm{n}
$$

Por otra parte, para la medición de la accesibilidad media integral se deben tener en cuenta los nodos de la red vial que pertenecen al área de estudio, es decir, la afectada por el CV, los cuales surtirán cómo origen de los viajes en el modelo ( $n$ ), mientras que la zona de la ciudad que recibe más viajes por parte de la zona de estudio se convertirá en el destino de los viajes del modelo $(\mathrm{m})$. Esta última variable se calcula teniendo en cuenta la matriz origen destino de la ciudad que se proporcionada por la administración municipal y que fue calculada durante el año 2017. Luego, se calcula el tvi entre los nodos determinados como origen y destino de los viajes mediante el método descrito en la accesibilidad media global y se obtiene el Tvi gracias a la ecuación 2, donde el número de nodos de origen $(n)$ y destino de viajes $(m)$ varía respecto a la ecuación 1.

$$
\overline{T_{v l j}}=\frac{\sum_{i=1}^{m} t_{v i j}}{m} \quad i=1,2,3, \ldots, n ; j=1,2,3, \ldots, \mathrm{m}
$$

Luego de obtener el Tvi para los distintos escenarios de análisis de accesibilidad media global e integral se procede a la construcción de las curvas isócronas de cobertura mediante el análisis geoestadístico a través del método Kriging ordinario con semivariograma lineal el cual ha mostrado un error menor y aproximaciones acertadas relacionadas a menor distancia entre los puntos a interpolar (Giraldo, 2002; Li y Heap, 2008). Este modelo geoestadístico puede ser ejecutado mediante software SIG o a través de la implementación de códigos de acceso libre en software cómo $\mathrm{R}$ o Python, lo cual permitirá que la investigación pueda ser replicada en otras regiones o ciudades. En este caso se utilizarán las herramientas geoestadísticas de ArcGis. Con las curvas isócronas de cobertura se realizarán comparaciones mediante la intersección de distintas capas de información socioeconómica de la zona de estudio tales cómo población, área, estratos, entre otras.

Etapa 4. Gradiente de ahorro. El gradiente de ahorro ( $G a)$ permite comparar los vectores de tiempo de viaje mínimo de cada escenario de accesibilidad media global e integral calculados, obteniendo que tan diferentes son los tiempos de la situación actual a con respecto la futura. En la ecuación 3 se observa cómo se realiza el cálculo, donde $A$ se refiere al vector promedio de viaje ya sea para la situación actual $(a)$ o futura $(f)$ (Moncada et al., 2018). Luego de obtener el vector de gradiente de ahorro se procede a ejecutar el modelo geo estadístico descrito en la etapa anterior para calcular las curvas isócronas de cobertura de ahorro.

$$
G a \%=\frac{A a-A f}{A a} * 100
$$

Etapa 5. Cálculo del índice de ahorro. Para el cálculo del índice de ahorro $(I A)$ se debe tener en cuenta el polígono de barrios del área de estudio (obtenido en la etapa 1) y las curvas isócronas de cobertura de ahorro obtenidas en la etapa anterior. Teniendo esto en cuenta se realiza una intersección entre dichos polígonos obteniendo el porcentaje de población (\%P) que se ve cubierto por cada porcentaje de ahorro generado (\%A). Luego, se realiza una ponderación entre el porcentaje de población y de ahorro y se realiza la sumatoria por barrios (j), encontrando los barrios del área de influencia que obtuvieron mayor beneficio por la construcción del proyecto de infraestructura vial (Ver ecuación 4).

$$
I A j=\sum_{i=1}^{n} \% A i * \% P i
$$




\section{RESULTADOS Y DISCUSIÓN}

A continuación, se presentan los principales resultados de la aplicación de la metodología propuesta en el caso de la "intersección La Carola" en Manizales, comenzando por las curvas isócronas de accesibilidad media global e integral, seguido por el gradiente de ahorro para ambas mediciones y terminando por enumerar el índice de ahorro para cada barrio estudiado.

Accesibilidad Media Global y Accesibilidad Media Integral. En la figura 3 se observan las curvas isócronas de cobertura de accesibilidad media global para la situación actual (izquierda) y futura (derecha) donde se puede ver claramente cómo la cobertura del intervalo de tiempos promedio de viaje más bajo (15,05 - 20 minutos) crece sobre el área de influencia, cubriendo más población. Asimismo, el tiempo promedio de viaje mínimo para la ciudad es cercano a los 15 minutos, lo cual es menor respecto a resultados previos, sin embargo, esto se debe a la calibración y cálculo de las penalidades por giro para el modelo de accesibilidad (Cardona, 2018).
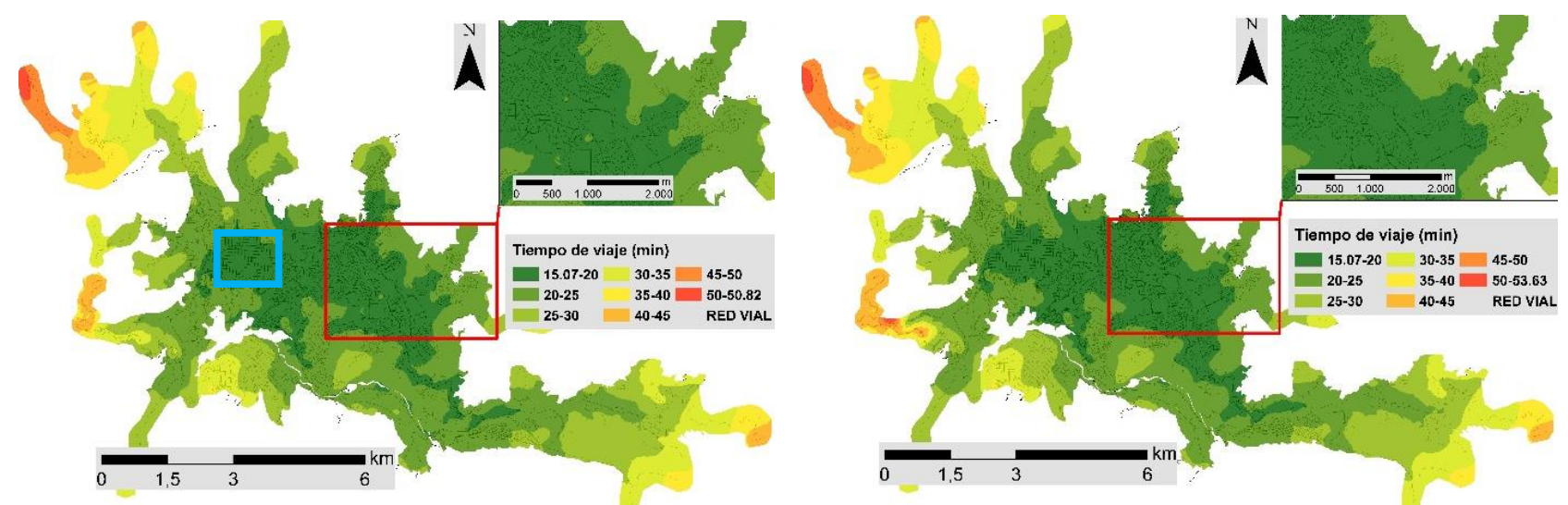

Fig. 3: Curvas isócronas, accesibilidad media global, situación actual (izquierda) y futura (derecha).

Por otra parte, en la figura 4 se observan las curvas isócronas de cobertura de accesibilidad media integral para la situación actual (izquierda) y futura (derecha), calculadas desde la zona de estudio hacia el centro de la ciudad teniendo en cuenta que el cálculo se realizó desde el área de estudio hacia el centro de la ciudad (ubicado dentro del cuadrado azul de la figura 3). En este caso, se registran tiempos de viaje mínimos de 8,27 minutos para la situación actual y de 7 minutos para la situación futura, lo que representa cerca de 1,5 minutos de ahorro en el viaje hacia el centro de la ciudad. Asimismo, se observa cómo la cobertura de las isócronas de tiempos de viaje de hasta 15 minutos crecen de la situación actual a la futura, generando mayor cobertura en menor tiempo para el área de estudio.
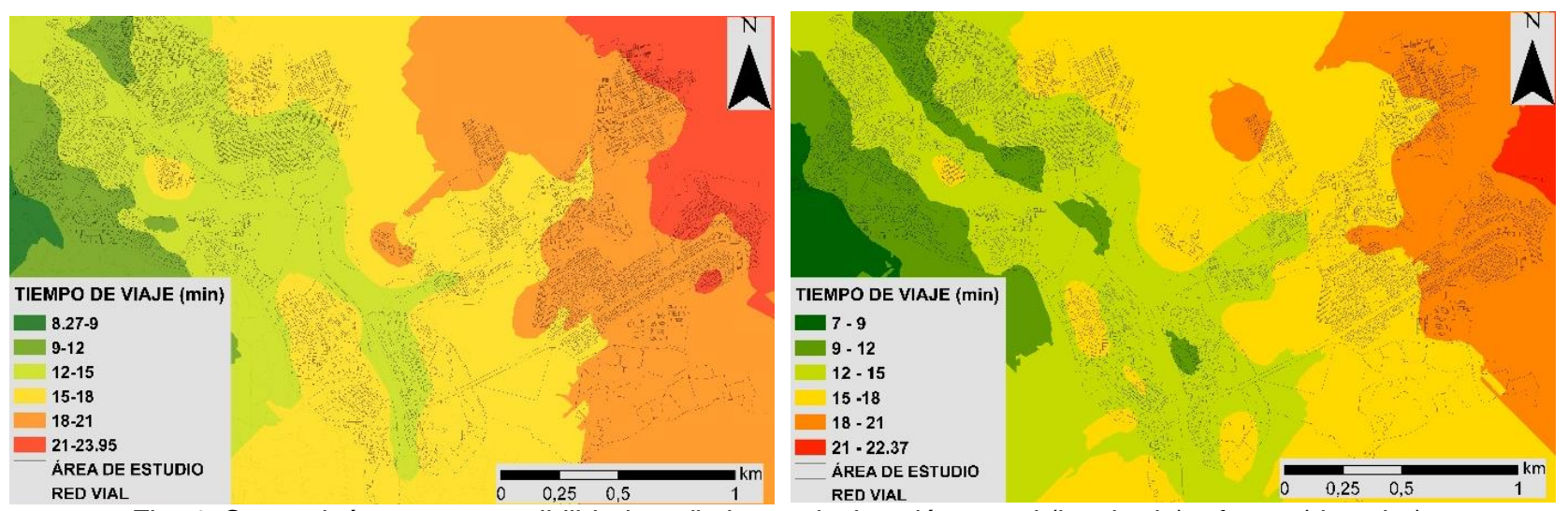

Fig. 4: Curvas isócronas, accesibilidad media integral, situación actual (izquierda) y futura (derecha).

En la figura 5 (izquierda), se presenta la ojiva de cobertura de población para las curvas isócronas de accesibilidad media global donde se evidencia un crecimiento cercano al $2 \%$ en la cobertura de población para los tiempos medios de viaje menores a 20 minutos, lo que representa más de 8.000 personas beneficiadas en toda la ciudad con la construcción de este proyecto. En los demás intervalos existe una diferencia en la cobertura de población, lo que a términos generales nos muestra que la obra de infraestructura genera beneficios en movilidad para los habitantes. En la figura 5 (derecha) se encuentra la comparación entre la cobertura generada por los dos escenarios de accesibilidad media integral medidos hacia el centro de la ciudad, evidenciándose que para cada intervalo de tiempo hay un mayor porcentaje de población en la situación futura. Citando algunos casos, en la situación actual se cubre un 40,3\% de la población (18.910 
habitantes) en tiempos menores a 15 minutos, mientras que para la situación futura crece hasta el $48,5 \%$ de la población (22.792 habitantes), un aumento que representa cerca de 4.000 personas que perciben un ahorro en su tiempo de viaje hacia el centro de la ciudad. Caso semejante para tiempos medios de viaje menores a 18 minutos, donde la cobertura aumenta cerca del 15\% entre ambos escenarios, lo que representa aproximadamente 8.000 personas directamente beneficiadas.
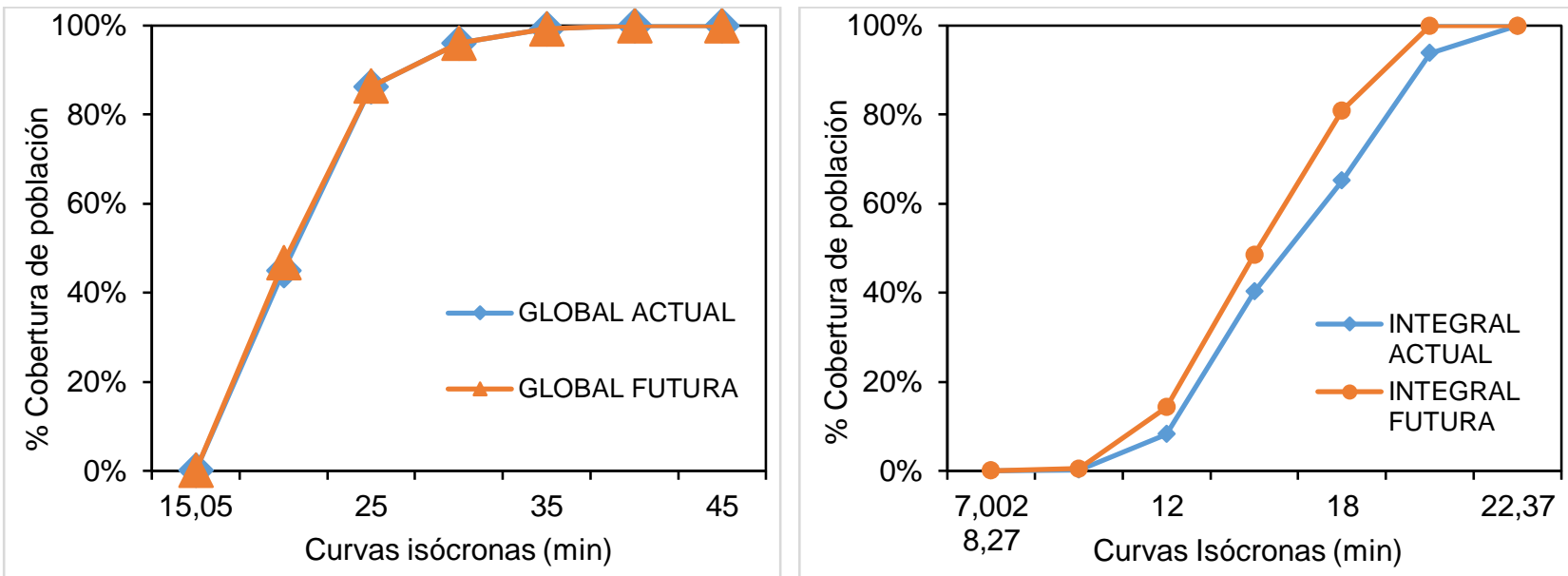

Fig. 5: Ojivas de cobertura de población, accesibilidad media integral (derecha) y accesibilidad media global (izquierda).

Gradiente de ahorro. Para la accesibilidad media global, se registran ahorros en el tiempo medio de viaje de hasta el $4,6 \%$ y unos ahorros notables sobre el área de influencia. Sin embargo, también ocurren aumentos en los tiempos de viaje promedio, impulsados por las condiciones de entrada del modelo de oferta donde se penalizan los giros. Sin embargo, cabe destacar que a nivel ciudad, cómo ya se mostró en el análisis anterior, la "intersección La Carola" genera un beneficio en la movilidad de los habitantes (ver figura 6 parte izquierda). Por otra parte, en el lado derecho de la figura 6 se observa el gradiente de ahorro dado para el análisis de accesibilidad media integral, donde se generan ahorros de hasta el $14 \%$ en el tiempo medio de viaje. En este caso también se observan algunas zonas donde no se obtuvo ahorro en el tiempo medio de viaje, sin embargo, estás no representan un gran porcentaje de la población.
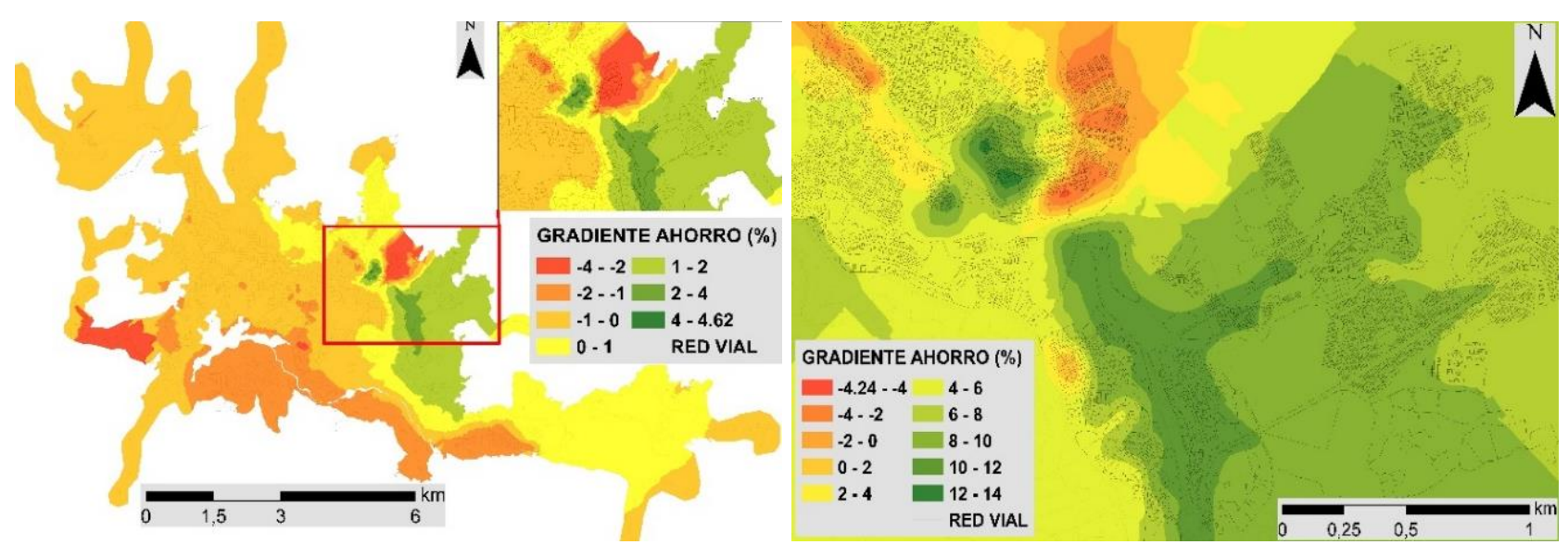

Fig. 6: Gradiente de ahorro, accesibilidad media global (izquierda) y accesibilidad media integral (derecha).

En este mismo sentido, en la figura 7 se observa el ahorro y el porcentaje de población en los barrios del área de influencia para la accesibilidad media integral. Barrios cómo La Baja Suiza y La Toscana (Ver Figura 1) alcanzaron hasta un $10 \%$ de ahorro en los tiempos medios de viaje para el $100 \%$ de su población. También, para estos barrios se obtuvo un ahorro de hasta el $12 \%$ para el $96 \%$ y $66 \%$ de la población, respectivamente, convirtiéndolos en las zonas que mayor beneficio presentan por la construcción del proyecto. Para porcentajes de hasta el $8 \%$ de ahorro, barrios cómo Bosques de Niza, La Cumbre, la Sultana, Minitas y Viveros (Ver Figura 1) ven beneficiada la totalidad de su población. El barrio La Carola, el cual está más cerca del proyecto, obtuvo ahorros de hasta el 14\% para el $3 \%$ de su población. Cabe destacar que en este barrio hubo un porcentaje de población que el modelo no alcanzo a captar en el ahorro percibido. A nivel general, el 5,7\% de la población percibe un ahorro de hasta el $12 \%$ en los tiempos de viaje, porcentaje que aumenta en $36,6 \%$ para un hasta $10 \%$ de ahorro. En general, cerca del $97 \%$ de la población del área de influencia obtiene ahorros en los tiempos de viaje de por lo menos el $2 \%$. 


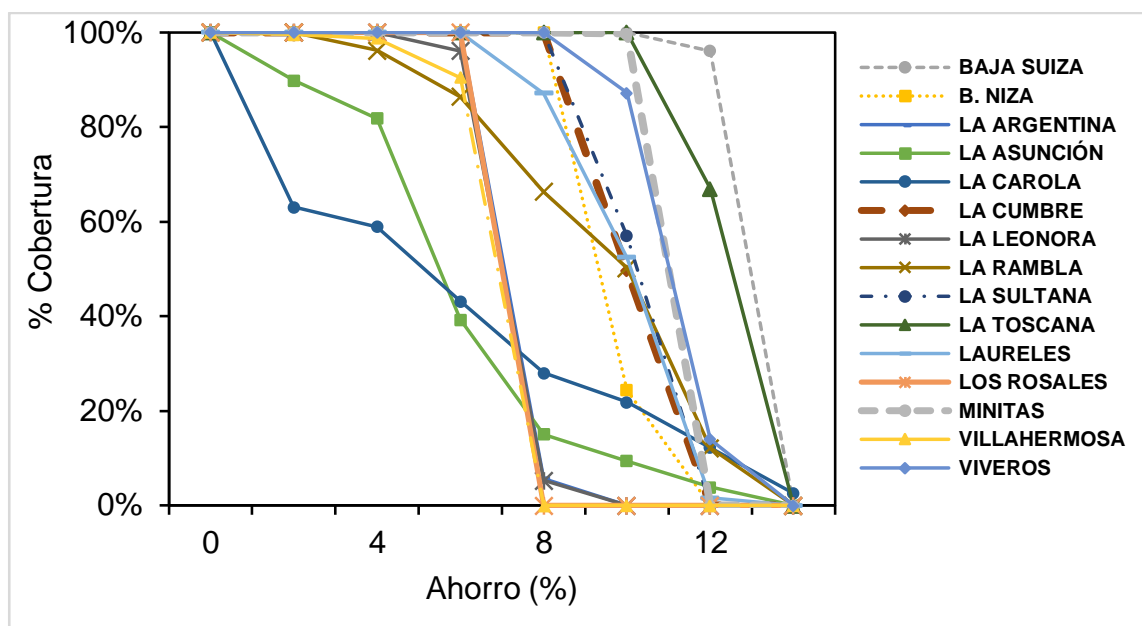

Fig. 7: Ahorro por barrio, accesibilidad media integral.

Índice de ahorro. El índice de ahorro (IA) calculado para cada uno de los barrios en ambos escenarios de accesibilidad se presenta en la tabla 1. En el caso del IA calculado teniendo en cuenta la accesibilidad media global (IA Global), el barrio de la Baja Suiza es el que posee un mayor IA con 2,50\%, seguido por el barrio la Toscana con 1,84\% y los barrios Bosques de Niza, La Cumbre y Minitas con 1,50\%. También se pueden observar diversos barrios que no registran ahorro (La Argentina, La Asunción, La Carola y La Leonora). En términos generales, la ciudad posee un IA del $0,25 \%$. Teniendo en cuenta estos resultados, se puede tener en cuenta el IA Global por barrio cómo variable que indique el ahorro comprobable que el proyecto generará en los tiempos de viaje del área de influencia.

Tabla 1: Cobertura por habitantes y área, accesibilidad media global - Situación Base

\begin{tabular}{|c|c|c|c|c|c|c|}
\hline Barrio & IA Global & IA Integral & \multirow[t]{9}{*}{$x x x$} & Barrio & IA Global & IA Integral \\
\hline Baja Suiza & $2,5 \%$ & $10,9 \%$ & & La Sultana & $1,5 \%$ & $8,1 \%$ \\
\hline Bosques De Niza & $1,5 \%$ & $7,5 \%$ & & La Toscana & $1,8 \%$ & $10,3 \%$ \\
\hline La Argentina & $0,0 \%$ & $5,1 \%$ & & Laureles & $1,7 \%$ & $7,8 \%$ \\
\hline La Asunción & $0,0 \%$ & $3,8 \%$ & & Los Rosales & $0,3 \%$ & $5,0 \%$ \\
\hline La Carola & $0,0 \%$ & $3,3 \%$ & & Minitas & $1,5 \%$ & $9,0 \%$ \\
\hline La Cumbre & $1,5 \%$ & $8,0 \%$ & & Villahermosa & $0,7 \%$ & $4,8 \%$ \\
\hline La Leonora & $0,0 \%$ & $5,0 \%$ & & Viveros & $1,3 \%$ & $9,0 \%$ \\
\hline La Rambla & $1,5 \%$ & $7,2 \%$ & & Total & $0,3 \%$ & $6,2 \%$ \\
\hline
\end{tabular}

Por otro lado, para el IA teniendo en cuenta los ahorros dados en los escenarios de accesibilidad media integral (IA Integral), los barrios poseen unos comportamientos similares, con el barrio la Baja Suiza con el mayor valor con un 10,9\% y el barrio La Toscana en segundo lugar con 10,3\% de IA Integral. El barrio que posee un menor IA es La Carola con 3,3\% lo cual es consecuente con los resultados de cobertura de población dados en ambos escenarios de accesibilidad. A nivel general, el área de estudio obtuvo un IA Integral de 6,2\% lo que nos indica que el intercambiador vial genera ahorros en el tiempo medio de viaje hacia el centro de la ciudad.

\section{DISCUSION FINAL}

Por medio de los modelos de oferta de transporte se pueden obtener los beneficios en movilidad y a los tiempos de viaje que generan nuevas obras de infraestructura vial. A través de la accesibilidad media global e integral se pudo comprobar el ahorro generado por la "Intersección La Carola" a nivel ciudad y en el área determinada por el INVAMA para el cobro por CV. El índice de ahorro (IA) medido en los barrios donde se ejecutó el cobro por CV muestra que existen algunos de ellos que obtienen unos beneficios mayores por la obra de infraestructura vial y que esto no está directamente relacionado con la cercanía a la misma debido a que se usaron modelos a nivel ciudad para medir los tiempos de viaje. Por ello, se recomienda usar micro simulaciones para complementar estos resultados y así medir los ahorros en tiempo medio de viaje que se generan a los barrios más cercanos a la obra propuesta para construcción. Está metodología complementa el método del doble avalúo usado por el INVAMA para determinar el área de influencia directa e indirecta, las cuales actualmente se definen de forma totalmente subjetiva y arbitraria, así como complementa la metodología para definir el monto de cobro por CV al momento de financiar una obra de infraestructura vial. Se recomienda a las regiones que pretenden financiar proyectos de infraestructura vial mediante el cobro por CV, utilizar el modelo Manizales, debido a que ha generado mayor aceptabilidad y satisfacción entre los 
contribuyentes. Asimismo, complementar su metodología mediante las variables abordadas en esta investigación. Para el cálculo de la matriz de tiempos de viajes entre pares origen y destino, actualmente existen diversos recursos en la web que permiten acceder a dichas variables de una forma precisa y rápida, haciendo que la metodología de investigación aquí presentada, sea fácilmente replicable en cualquier región o ciudad donde se estén llevando a cabo evaluaciones de proyectos financiados mediante dicha metodología, incluyendo contextos donde hagan falta algunas de sus herramientas básicas (software, red vial, etc.) (Lahoorpoor y Levinson, 2020).

\section{CONCLUSIONES}

De acuerdo al trabajo presentado y a los resultados obtenidos, se pueden plantear las siguientes conclusiones principales:

1. Los análisis de accesibilidad media global y de gradiente de ahorro son variables fundamentales que deben ser establecidas al momento de realizar la evaluación del beneficio que generará un proyecto sobre una región determinada, reforzando así la definición del área de influencia directa e indirecta sobre la que debería realizarse el recaudo de $\mathrm{CV}$, teniendo en cuenta el porcentaje de ahorro en los tiempos medios de viaje generados, pues en la actualidad, la definición del área de influencia es totalmente subjetiva.

2. El método del doble avalúo abordado por el modelo Manizales debe ser complementado con las variables Índice de Ahorro Global e Índice de Ahorro Integral, ambas calculadas por barrio o zona censal en el área de influencia directa e indirecta, con el fin de tener un mayor detalle en el momento de la asignación del beneficio dado por el proyecto a construirse.

3. En el caso del Índice de Ahorro Global es necesario utilizarlo al momento de medir los ahorros en tiempos medios de viaje generados por la construcción de la obra, al comparar los escenarios actual y futuro, lo cual permitirá obtener de forma más detallada los ahorros según barrio o zona censal, en base a tiempos y nos sólo a distancia, como actualmente ocurre. Por otra parte, el Índice de Ahorro Integral es necesario utilizarlos al momento de calcular la mejora en el acceso a áreas comerciales ya definidas, para lo cual es de vital importancia constatar dicha relación con la matriz origen destino o con la aplicación de encuestas de preferencias declaradas, definiendo con más exactitud la zona de la ciudad hacia donde más viajan las personas del área de influencia del proyecto al cual se le cobrará CV.

4. Está metodología permite cuantificar el porcentaje de ahorro de un proyecto mediante modelos de accesibilidad por lo que generará una mejora considerable a la metodología de doble avalúo utilizada por el modelo Manizales debido a que las variables movilidad podrán ser calculadas por zonas o barrios, detallando aún más el cálculo de la CV. Asimismo, estos modelos pueden determinar que los proyectos no generen ahorros o generen perdidas en tiempo medio de viaje en algunos sectores por lo que, a estos contribuyentes, según la fórmula de asignación del recaudo, se les debería cobrar un menor valor que a aquellos contribuyentes que realmente tendrían ahorro en el tiempo de sus desplazamientos dada la construcción del proyecto.

\section{AGRADECIMIENTOS}

Los autores agradecen al Grupo de Investigación en Movilidad Sostenible, adscrito a la Facultad de Ingeniería y Arquitectura del Departamento de Ingeniería Civil de la Universidad Nacional de Colombia Sede Manizales.

\section{REFERENCIAS}

Añez, J., De La Barra, T. y Pérez, B., Dual graph representation of transport networks, doi.org/10.1016/01912615(95)00024-0, Transportation Research Part B: Methodological, 30(3), 209-216 (1996).

Borrero, O., Betterment Levy in Colombia: Revelance, procedures, and social acceptability, Land Lines, Lincoln Institute of Land Policy, 23(2), 14-19 (2011).

Borrero, O., Contribución de valorización o mejoras en Colombia. Análisis de la experiencia colombiana, Lincoln Institute of Land Policy, 1-44 (2013).

Caldwell, T., On Finding Minimum Routes in a Network With Turn Penalties, doi.org/10.1145/366105.366184, Communications of the ACM, 4(2), 107-108 (1961).

Cardona, S., Propuesta metodológica para el cálculo de las penalidades por giro en modelos de accesibilidad, Tesis de Maestría, Universidad Nacional de Colombia, Manizales, Colombia (2018).

Delling, D., Sanders, P., Schultes, D. y Wagner, D., Engineering route planning algorithms, doi.org/10.1007/978-3-64202094-0_7, En: Lerner J., Wagner D., Zweig K.A. (eds) Algorithmics of Large and Complex Networks. Lecture Notes in Computer Science, vol 5515. Springer, Berlin, Heidelberg (2009). 
Dijkstra, E.W., A note on two problems in connexion with graphs, doi.org/10.1007/BF01386390, Numerische Mathematik, 1(1), 269-271 (1959).

Escobar, D.A. y García, F.J., Análisis de priorización de proyectos viales, Caso Manizales (Colombia), $1^{\mathrm{a}}$ edición, Universidad Nacional de Colombia, Manizales, Colombia (2012).

Fernández, A., La contribución de valorización en Colombia, ISBN: 84-8272-170-4, 2ª Edición, Editorial Temis Librería, Bogotá, Colombia (1981).

Geurs, K.T. y Van Wee, B., Accessibility evaluation of land-use and transport strategies: Review and research directions, doi.org/10.1016/j.jtrangeo.2003.10.005., Journal of Transport Geography, 12(2), 127-140 (2004).

Giraldo, R. Introducción a la geo estadística. Teoría y aplicación, Universidad Nacional de Colombia, Facultad de Ciencias, Departamento de Estadística, Bogotá, Colombia (2002).

Guzman, L.A., Oviedo, D. y Cardona, R., Accessibility Changes: Analysis of the Integrated Public Transport System of Bogotá, doi.org/10.3390/su10113958, Sustainability, 10(11), 3958 (2018).

Hansen, W.G., How Accessibility Shapes Land Use, doi:10.1080/01944365908978307, Journal American Institute of Planners, 25(2), 73-76 (1959)

Ingram, D.R., The Concept of Accessibility: A search for an operational form, doi: 10.1080/09595237100185131, Regional Studies, 5(2), 101-107 (1971).

INVAMA - Instituto de Valorización de Manizales, Detalle del proceso: INV-L-001-2017. Manizales, Colombia (2017).

Lahoorpoor, B. y Levinson, D.M., The Transit Travel Time Machine: Comparing Three Different Tools for Travel Time Estimation, Transportation Research Board Conference, Washington, DC, January (2020).

Li, J. y Heap, A.D., A Review of Spatial Interpolation Methods for Environmental Scientists, ISSN 1448-2177, Geoscience Australia, Record 2008/23, 137 pp (2008).

Liu, S. y Zhu, X., An integrated GIS approach to accessibility analysis, doi.org/10.1111/j.1467-9671.2004.00167.x, Transactions in GIS, 8(1), 45-62 (2004).

Miramontes, E., Vidaña, J. y Rodríguez, A., Análisis y Evaluación de Intersecciones Urbanas, CULCyT: Cultura Científica y Tecnológica, ISSN-e 2007-0411, 12(56), 51-60 (2015).

Moncada, C.A., Cardona, S. y Escobar, D.A., Saving Travel Time as an Urban Planning Instrument. Case Study: Manizales, Colombia, doi: 10.5539/mas.v12n6p44, Modern Applied Science, 12(6), 44-57 (2018).

Navarro, E. La contribución por valorización como mecanismo de financiación de obras públicas, doi: https://doi.org/10.32997/2256-2796-vol.8-num.16-2016-1532, Revista Jurídica Mario Alario D’Filippo, 8(16), 67-84 (2016).

Ortuzar, J.D. y Willumsen, L.G., Modelling Transport, ISBN: 978-0-470-76039-0, 4ª Edición, John Wiley \& sons (2011).

Oviedo, D., Scholl, L., Innao, M. y Pedraza, L., Do Bus Rapid Transit Systems improve accessibility to job opportunities for the poor? The case of Lima, Peru, doi.org/10.20944/preprints201903.0150.v1, Sustainability, 10, 1-27 (2019).

Owens, S., From "predict and provide" to "predict and prevent"?: Pricing and planning in transport policy, doi.org/10.1016/0967-070X(95)93245-T, Transport Policy, 2(1), $43-49$ (1995).

Pirie, G.H., Measuring Accessibility: A Review and Proposal, doi.org/10.1068/a110299, Environment and Planning A, 11(3), 299-312 (1979).

Smolka, M., A New Look at Value Capture in Latin America, Land Lines, Lincoln Institute of Land Policy, 24(3), 10-15 (2012).

Talen, E., y Anselin, L., Assessing spatial equity: An evaluation of measures of accessibility to public playgrounds, doi.org/10.1068/a300595, Environment and Planning A, 30(4), 595-613 (1998).

Walsh, S., Cullinan, J. y Flannery, D., The Impact of Proposed Higher Education Reforms on Geographic Accessibility to Universities in Ireland, doi.org/10.1007/s12061-016-9193-3, Applied Spatial Analysis and Policy, 10(4), 515-536 (2017).

Weber, J., The properties of topological network connectivity measures and their application to U.S. urban freeway networks, doi.org/10.1080/00330124.2015.1106324, Professional Geographer, 68(3), 485-495 (2016).

Yiannakoulias, N., Bland, W. y Svenson, L.W., Estimating the effect of turn penalties and traffic congestion on measuring spatial accessibility to primary health care, doi.org/10.1016/j.apgeog.2012.12.003, Applied Geography, 39, 172-182 (2013). 